\title{
International symposium on the formation and development of the Chinese nation with multi-ethnic groups_to commemorate the 30th anniversary of the publication of Fei Xiaotong's theory of the Chinese nation with multi-ethnic groups
}

\section{Haitao Liu}

Received: 31 July 2018 / Accepted: 7 September 2018 / Published online: 01 October 2018 (C) The Author(s). 2018 Open Access This article is distributed under the terms of the Creative Commons Attribution 4.0 International License (http://creativecommons.org/licenses/by/4.0/), which permits unrestricted use, distribution, and reproduction in any medium, provided you give appropriate credit to the original author(s) and the source, provide a link to the Creative Commons license, and indicate if changes were made.

\begin{abstract}
A Summary of International Symposium on the Formation and Development of the Chinese Nation with Multi-ethnic Groups in order to Commemorate the 30th Anniversary of the Publication of Fei Xiaotong's Theory of the Chinese Nation with Multi-ethnic Groups.
\end{abstract}

Keywords Summary $\cdot$ Symposium $\cdot$ Chinese nation $\cdot$ Multi-ethnic groups

\section{Main text}

The Chinese nation is a national community formed with rich connotations based on long-term exchanges and interactions. It has been sublimated from a free entity into a self-conscious entity through time-honored processes of national fusion and cultural communication and especially through the anti-imperialist, anti-feudal, and anti-invasion struggles in which the Chinese people of all ethnicities were united. In the course of development, the Chinese nation has gradually reached a pluralistic unity, a pattern in which plural ethnic groups form a united nation. Understanding this, Fei Xiaotong published the paper "The Chinese Nation with Multi-ethnic Groups" in 1988 in which he provided a macro theoretical summary of the formation and structural characteristics of the Chinese nation. Fei Xiaotong's theory is a

H. Liu $(\square)$

Editorial Office of Ethno-National Studies, Institute of Ethnology and Anthropology, Chinese Academy of Social Sciences, Building 6, No. 27, Zhongguancun South Avenue, Beijing 100081, China e-mail: liuhaitaozuoye@ 126.com 
reasoned explanation of the relationship between nation and state in the formation and development of a unified multi-ethnic country. It has drawn widespread attention from various circles in China and abroad, especially academic circles, and injected an impetus to the study of the Chinese nation.

Since the introduction of this theory, new problems and trends have arisen in ethnic relations and developments in changing domestic and international contexts have raised new requirements for ethnic studies. Being faced with the requirements for ethnic studies and ethnic work in the new era, the CASS Forum to celebrate the 60th anniversary of the establishment of the Institute of Ethnology and Anthropology of Chinese Academy of Social Sciences was held on the occasion to commemorate the 30th anniversary of the publication of Fei Xiaotong's theory of the Chinese Nation with Multi-ethnic Groups. It conducted in-depth systematic academic discussions on the formation and development of pluralistic unity in multiple dimensions, such as ethnic theory, ethnic history, ethnology and anthropology, and world nationalities, contributing to the central task of "creating a strong sense of community for the Chinese nation" put forward by the 19th National Congress of the Communist Party of China (CPC) and to the rapid move to develop systems of philosophy and social sciences and discourses with Chinese characteristics.

The Forum was sponsored by the Chinese Academy of Social Sciences (CASS) and organized by the Institute of Ethnology and Anthropology (IEA) and the Bureau of International Cooperation (BIC) with the assistance of the China Federation of Societies of Ethnic Studies, Chinese Association of Ethnic Theory Research, Association of Chinese Ethnology, Chinese Association for Nationality History, the Editorial Office of Ethno-National Studies, and the Editorial Office of World Ethno-National Studies. It took place on April 13-15, 2018 in Beijing, and received over 80 papers by more than 100 experts, scholars, and journalists from colleges and universities, research institutes, and news media in China and abroad, including L.N. Gumilyov Eurasian National University, CASS, the Central Party School, State Ethnic Affairs Commission (SEAC), China Tibetology Research Center, Chinese National Academy of Arts, Academia Sinica, Peking University, Nanjing University, Tsinghua University, Minzu University of China Kazakhstan, Sun Yat-sen University, Xiamen University, Renmin University of China, Sichuan University, Shandong University, Ningxia University, Jilin University, Anhui University, Southwestern University, Lanzhou University, Jinan University, South-Central University for Nationalities, Northwest University for Nationalities, Guangxi University for Nationalities, Yunnan Nationalities University, Qinghai Nationalities University, Tianjin Normal University, Shaanxi Normal University, Shanghai Normal University, Northeast Normal University, East China Normal University, Inner Mongolia University of Finance and Economics, Zhejiang University of Finance \& Economics, Nanjing University of Chinese Medicine, Jishou University, Hubei University, Hebei Academy of Social Sciences, Social Sciences Academic Press, Intellectual Property Publishing House, and China Ethnic News.

At the opening ceremony, Prof. Li Peilin, CASS Vice President, made the first address, fully affirming the theoretical value and practical significance of this Forum in consideration of President Xi Jinping's requirements for national fusion and unity in the new era and the origin and contribution of Fei Xiaotong's theory. Zhu Weiqun, 
former Executive Deputy Director of the United Front Work Department of the CPC Central Committee and former Head of the Committee for Ethnic and Religious Affairs of the National Committee of the Chinese People's Political Consultative Conference (CPPCC), stressed the practical significance of Fei's theory. Du Rongkun, Honorary CASS Member and former IEA Director and Research Fellow, highlighted Fei's contribution to the study on Chinese nationality history. Wang Weimin, Vice Chairman of the Counsellor's Office of the State Council (COSC), recalled Fei's many achievements and outstanding contributions in the field of ethnic studies. Zhang Junhao, Deputy Director of the SEAC Research Center for Ethnic Theory and Policy (Ethnic Issues Research Center), emphasized the academic and practical significance of Fei's theory and wished on behalf of SEAC the Forum a complete success. In addition, Kwang Ok Kim, Korean anthropologist and Distinguished Professor of Shandong University, delivered an address on behalf of the participants. He recognized that Fei Xiaotong's theory is built on the investigation of national development processes and spoke highly of Fei's learning philosophy of service for the people. Zhang Zhe, Deputy Secretary General of the COSC Social Investigation Center, provided a systematic review of Fei's life stories and academic contributions in the address on behalf of his family. Wang Yanzhong, IEA Director and Research Fellow, recalled the innovative development of President Xi Jinping's ideas for ethnic work in the new era and emphasized the theoretic and practical significance of creating a strong sense of community for the Chinese nation and building a Chinese national community. The opening ceremony was chaired by Wang Lei, BIC(Bureau International Cooperation, CASS)Director.

Keynote speeches and four sessions came after the opening ceremony. In keynote speeches, Prof. Shi Jinbo, CASS Member, elaborated on the formation background and contemporary values of the theory of pluralistic unity. Prof. Chen Yuning of Ningxia University stressed that the ability to unite gives a significant impetus to the great rejuvenation of the Chinese nation. Prof. Ma Yan from Peking University noted that Fei Xiaotong's ethnic research and exploration is constantly being evolved and deepened. Prof. Hua Tao of Nanjing University demonstrated Fei's reflection on the debate about "There Is Only One Chinese Nation," covering such issues as ethnic identification, ethnic formation and ethnic essence, and Fei's rethinking on reflection, and he emphasized the significance of such reflection and rethinking for building the power to unite the Chinese nation. Prof. Chi-na Wu of Academia Sinica discussed Chinese nation building from the perspective of the rheology of ethnic political structures. Prof. Xu Ping of the Central Party School talked about the significance of the pluralistic-unity theory for the great rejuvenation of the Chinese nation. IEA Research Fellow Wang Xien believed that the pluralistic-unity theory can be upgraded to pluralistic unity, a generalization of the structure of both the Chinese nation and the world's nations that abandons cultural relativism and transcends multiculturalism. Baha,Research Fellow of L.N. Gumilyov Eurasian National University of Kazakhstan, expounded the Qing Dynasty's ideas and their impact on Kazakh ruling legitimism through a case of Qing-Kazakh exchanges during the 48th year of Emperor Qianlong's reign. Prof. Zhao Shugang of Anhui University, also a Taiwan scholar, analyzed Taiwan's past and current identification and memory of the Chinese nation. Prof. Ma Guoqing of Minzu University of 
China revealed the connotation and significance of the change from the pluralistic unity of the Chinese nation to a community of a shared future for mankind. The keynote speeches were co-chaired by Wang Yanzhong and Shi Jinbo.

1. Session on Commemoration of Fei Xiaotong. Prof. Yang Wenjiong of Lanzhou University believed that Fei's theory has modern value as a Chinese paradigm for understanding the modern nation-state. Prof. Zhang Yun of China Tibetology Research Center, summarized the characteristics of Fei's theory. Prof. Fang Lili of the Chinese National Academy of Arts pointed out that the road of a community with a shared future depends on pluralistic unity and mutual appreciation. Li Dalong of the CASS Research Center for Chinese Borderland History and Geography (RCCBHG) revealed through research Fei's important contributions in explaining the theory of the Chinese nation. Prof. Zhang Dongning of Northeast Normal University explained the pluralistic and united community of humanity's shared future under the perspective of international society. Su Hang, IEA Associate Research Fellow, discussed Fei Xiaotong's ideas on nation and state. Prof. Huang Baiquan of Hubei University highlighted Fei's contribution to the study of the Wuling Ethnic Corridor. Song Peijun, RCCBHG senior editor, explained Fei's theory of pluralistic unity based on the practices resulting from the late Qing, early Republic policy of Five Races Under One Union. Liu Bo'er, lecturer of Nanjing University of Chinese Medicine, examined in retrospect the theoretic design of pluralism and unity in national circles during the Republic of China. This session was chaired by Prof. Xu Ping and reviewed by Se Yin.

2. Session on Ethnic Theory. Prof. Kong Ting from Zaozhuang University discussed the creation of a strong sense of community for the Chinese nation in the new era, and Prof. Zhang Sanna of Tianjin Normal University expounded the localization of Marxist national theory in China based on the "two-community" idea. Prof. Jin Zhiyuan of Xi'an International Studies University understood the pluralistic-unity structure of the Chinese nation from the perspective of the elimination of the two nationalisms. Wang Qichang, lecturer at Inner Mongolia University of Finance and Economics, explored and analyzed the deepened development of Xi Jinping's theory of the pluralistic unity of the Chinese nation. Prof. Zhao Gang of Yanbian University interpreted the political attributes of the sense of community for the Chinese nation. Prof. Chang Shiyin of Tianjin Normal University talked about spiritual home construction and multicultural governance from the perspective of the pluralistic unity of contemporary China. Prof. Yan Qing of Minzu University of China discussed Chinese descriptions and interpretations of national and state structures, and Prof. Wang Jun presented nation and nation building at issue in the aspects of connotation, path and evaluation. Prof. Li Zhanrong from Zhejiang University of Finance \& Economics elaborated the legal significance of writing "Chinese nation" into the constitution. Dr. Jiao Xuezhen of Minzu University of China considered that there is a shift from dignity protection to mutual respect in the pattern of contemporary ethnic relations. IEA Research Fellow Liu Hong and Dr. Zhang Yuxuan of Minzu University of China discussed the plight of European nation-states and issues of building a community based on the shared future for mankind. Dr. Deng Xiao of the Central Party School talked about cultural awareness and self-confidence in the era of the Internet. Yin Hao, lecturer at the Party School of Inner Mongolia Autonomous 
Region, shared ideas on the consideration and pathway for the inheritance and education of outstanding traditional cultures of less populous ethnic groups in the context of pluralistic unity. QidongBailig, Associate Professor of Northwest University for Nationalities, discussed the globally pluralistic and united pattern of humans formed on the basis of humanistic choices. Prof. TaoDaoqiang, Associate Professor of Zaozhuang University, probed into the diversity of the Han nationality in the context of China's pluralistic unity. Dr. Benjamin Miles of Nanjing University examined the modern shortage of the pluralistic-unity theory. Huang Zhaoyu, Research Fellow of China Institute of Contemporary International Relations, discussed the issue of reshaping the community of major ethnic groups under the global trend. The session was chaired by Liu Hong and Prof. Ding Hong respectively and reviewed by Wang Xi'en and Prof. Wang Jun.

3. Session on Nationality History. Prof. Cheng Nina of Jilin University commented on Zhang Boquan's theory of a united China. Prof. Luo Kanglong of Jishou University, illustrated studies on cultural integration in the Chinese nation, focusing on education and governance. Prof. Ding Hong of Minzu University of China examined the pluralism and unity of Chinese culture from the perspective of ethnic Chinese overseas. Shao Lei from IEA investigated the development of Chinese history from the perspective of northern ethnic groups, focusing on the rise of five ethnic minorities. Prof. Li Donghong of Yunnan University revealed the significance of the Bai ethnic group in the formation and development of pluralistic unity in China in the aspects of local foundations, local traditions and citizens. Prof. Tian Min of South-Central University for Nationalities interpreted the historical attributes of China's pluralistic unity from the viewpoint of the national unity and state identity of southern ethnic minorities. Prof. Cang Ming of Minzu University of China illustrated the outlooks on state and nation indicated in the Picture Album of Subordinate Peoples of the Qing Dynasty. IEA Research Fellow Mu Shihua analyzed the literature of multi-ethnic Chinese character compounds and the evolution of the pluralistic-unity pattern in China. He Wei, a lecturer in Dali University discussed the expansion of state borders and establishment of provincial governors by Emperor Wu of the Han Dynasty. IEA Research Fellow Peng Fengwen conducted a historical investigation of the path to the Chinese national community based on research into the state construction and political transformation of the Northern Wei Dynasty. Prof. Liu Zhiwen of Tonghua Normal University made clear the course of the Korean ethnic group's integration into the Chinese national community, including refugees, immigrants and nationals. Dr. Shang Qing of Minzu University of China studied the issue of national identity under state administration and historical legacy from an interdisciplinary perspective. Prof. PengFurong of Yangtze Normal University discussed the game between submission to empire and the interests of tusi (literally earth lords) through a case of Wang Yangming's three books on Xishui, while RCCBHG Research Fellow Wang Kangyi described the jimi (control) prefectures, decrees and laws of the Tang Dynasty and revealed that the governance had been accustomed and unified. Prof. Wei Zhijiang of Sun Yat-sen University deliberated over the relationship between the Liao Empire and the Inner Asian countries and the international orderof the region. Li Chunxi, lecturer at Jinan University, shared views on YelvChucai's identity crisis and cultural identity. The session was chaired by 
Prof. Wei Zhijiang and PengFengwen respectively and reviewed by Prof. Shen Weirong and Li Dalong.

4. Session on Ethnology and Anthropology. Prof. Wang Mingming of Peking University interpreted Fei Xiaotong's thought of pluralistic unity from the perspective of a civilized or super-social system. He believed that China with pluralistic unity cannot be simply summed up by "society" but a "society in society." Prof. Zhao Xudong of Renmin University of China expounded Fei Xiaotong's thought of pluralistic unity by investigating crowd relationships, a basic issue of anthropology, from the viewpoint of one-to-many relationship in the countryside, nation, and the world. He held that in the context of global cultural transformation, the one-to-many issues in cultural, social, and individual dimensions once again deserve attention and should be addressed. Prof. Gao Bingzhong of Peking University adopted the perspective of pluralistic unity to study the transition from the Chinese nation to the world community, and argued that the modern concept of nation, no matter under what circumstances, relies on the concept of world society. In this respect, the look into the evolution from the theory of pluralistic unity to the theory of cultural consciousness found that Fei Xiaotong's methodology runs through global consciousness. Prof. Liu Neng from Peking University provided a temporal-spatial analysis of Fei Xiaotong's concepts of pluralistic unity and unity of the Chinese nation, revealed that social and cultural conditions that contribute to the unity of the Chinese nation under the contemporary context, and considered that theoretically, the social mechanisms promoting pluralistic unity are themselves pluralistic and that contemporary unity has a complex context and multiple social foundations.

Based on the temporal-spatial analysis of The Pattern of Pluralistic Unity of the Chinese People authored by Fei Xiaotong, Associate Professor Liu Qian of Renmin University of China interpreted the spiritual pattern of the new era from the perspective of pluralistic unity and shared his extended thinking on pluralistic unity which regarded such a spiritual pattern and cultural quality as a renewal and conscious inheritance of Chinese cultural traditions in contemporary times. IEA Research Fellow Zhang Jijiao raised the viewpoint of new nationalism based on pluralistic unity and the multi-ethnic state in an attempt to promote Fei Xiaotong's academic thought. Prof. Jia Zhongyi of Minzu University of China creatively interpreted the pluralistic unity of the Chinese nation in ethnic, regional, economic \& cultural, and religious dimensions based on field surveys and his own experiences. The thesis of Prof. Gao Xiangdong and Dr. Wang Xinxian of East China Normal University showed changes in the spatial composition and geographical pattern of China's ethnic minorities from a macro statistical perspective based on the analysis of spatial distribution and demographic center-of-gravity shift from 1953 to 2010. The revealed characteristics of pluralism and its changes provided empirical evidence for Fei Xiaotong's theory. Prof. Pu Lichun of Yunnan Nationalities University discussed the inheritance of Lisu language under the pattern of pluralistic unity based on the investigation of Lu Madeng Township in Fugong County, Nujiang Prefecture, Yunnan Province. Prof. Yuan Dongsheng of Guiyang University conducted a multi-dimensional probe into the interactive and reciprocal inter-ethnic relations in economic activities based on field investigation into a Shui ethnic village. IEA Research Fellow Wang Jianfeng illustrated the practical significance of Ganzi 
Tibetan Autonomous Prefecture's efforts to strengthen ethnic interactions and exchanges and promote ethnic unity and progress. Associate Professor Wang Lihong of Ningxia University, discussed the education and research of ethnic unity from the perspective of key ethnic signs through a case of the Primary School Affiliated with Guangxi Teachers Education University. Prof. Sun Jiuxia of Sun Yat-sen University revealed the impact of tourism flow on the pattern of pluralistic unity of the Chinese nation in the new era. With the theory of pluralistic unity as a guide, Prof. Fu Qianji of Northwest University for Nationalities explored the development of Tibetan-Han bilingual education in Gannan Tibetan Prefecture. Bao Laijun from Hebei Academy of Social Sciences talked about the spread of the concept of pluralistic unity demonstrated by a Xinjiang-themed documentary on the Belt and Road Initiative. The session was chaired by Prof. Liu Zhengyin and Prof. Fang Lili respectively and reviewed by GuHaiyan and Prof. Fang Lili.

During the meeting, other scholars also shared their opinions on related issues. Throughout the meeting, climax continued owing to the brilliant presentations, reports, and related comments, responses, and discussions of the participating experts. At the closing ceremony, Se Yin, Chang Shiyin, Peng Furong, and Liu Haitao wrapped up the four sessions respectively, and Wang Yanzhong delivered concluding remarks on the meeting. The Forum exhibited the prominent characteristics of close relevance to the theme of the times, rich content, wide disciplinary vision, and extensive representation of participants. Fei Xiaotong's theory of pluralistic unity of the Chinese nation, with historical penetrating power, retains its important significance as theoretical guidance for "creating a strong sense of community for the Chinese nation" in the new era. On behalf of IEA, Wang Yanzhong expressed thanks to all the participating scholars and staff. The closing ceremony was chaired by Liu Zhengyin.

\section{Authors' contributions}

The author read and approved the final manuscript.

\section{Competing interests}

The author declares that he/she has no competing interests.

\section{Publisher's Note}

Springer Nature remains neutral with regard to jurisdictional claims in published maps and institutional affiliations. 